\title{
B-chromsomes in Sorghum stipoideum
}

\author{
TA-PEN WU \\ Department of Botany, National Taiwan University, Taipei, Taiwan, Republic of China
}

\begin{abstract}
It is clear from pachytene analysis that the B-chromosome of Sorghum stipoideum is a euchromatic iso-chromosome which has probably originated from centromere misdivision of one of the A-chromosomes. B-bivalents behave in a more stable and regular fashion at meiosis than do B-univalents. These B-univalents frequently lag and divide precociously during male meiosis. Mosaicism of B-chromosomes is found in microsporocytes and tapetal cells, while they are totally eliminated from stems and leaves.
\end{abstract}

Keywords: B-chromosomes, elimination of B-chromosomes, mosaicism of B-chromosomes, pachytene analysis, Sorghum stipoideum.

\section{Introduction}

B-chromosomes have been reported in numerous plant and animal taxa (Jones \& Rees, 1982). The origin and cytological behaviour of B-chromosomes in Sorghum nitidum and $S$. purpureosericeum have been described previously (Wu, 1980, 1984). This paper deals with the cytological behaviour of B-chromosomes which have been found in another Sorghum species, S. stipoideum. The pachytene karyotype of the plant with B-chromosomes was also analysed in order to compare the morphology and relationship between A- and B-chromosomes in this species.

\section{Materials and methods}

A single 2B plant of Sorghum stipoideum was grown from seeds supplied by the Division of Plant Industry, CSIRO, Australia. The present study was made using 14 progeny produced by the $2 \mathrm{~B}$ female parent. Young spikes were fixed in 3:1 ethanol acetic acid for $24 \mathrm{~h}$ for cytological analysis and then stored in 70 per cent ethanol. Meiosis in microsporocytes and somatic mitosis in anther walls and meristematic tissues were studied by using the propiono-carmine squash technique. Observations and photomicrographs were made using temporary slides.

At the pachytene stage, measurements of chromosome and heterochromatic lengths were made on nine cells in which all A- and B-chromosomes could be followed from end-to-end and the positions of all centromeres were discernible. Measurements of lengths and positions of chromosome landmarks were also obtained from identifiable pachytene chromosomes of other cells, as individual chromosome landmarks were not visible in every cell.

\section{Results}

\section{Pachytene chromosome morphology}

The synapsis of homologous A-chromosomes at pachytene was complete and normal in Sorghum stipodeum. Typical pachytene A-chromosome bivalents had darkly staining heterochromatic regions on both sides of the centromere and light staining distal euchromatic regions. As the appearance and length of the chromosomes varies constantly during pachytene, it was difficult to identify individual chromosomes based only on the chromosome lengths and the arm ratios. However, each chromosome exhibited a definite pattern involving features such as length and position of heterochromatic regions, position of deeply staining knobs, chromomeres and nucleolus organizers which made it possible to identify individual chromosomes. Of the five A-bivalents at the pachytene stage, chromosome 1 (Figs $1 \mathrm{a}$ and $\mathrm{b}$ and 2 ) is the most asymmetrical. Its short arm is entirely heterochromatic. These conspicuous features make it easily distinguishable from the rest of the complement. In this species, chromosome 4 (Figs 1a, b, and e and 2) is the main nucleolusorganizing chromosome. The nucleolus is formed on the short arm close to the centromere. Chromosome 3 (Figs 1a, b, e and 2) occasionally participates in organizing the nucleolus, and its nucleolus organizer is located at a subterminal position on the short arm. 


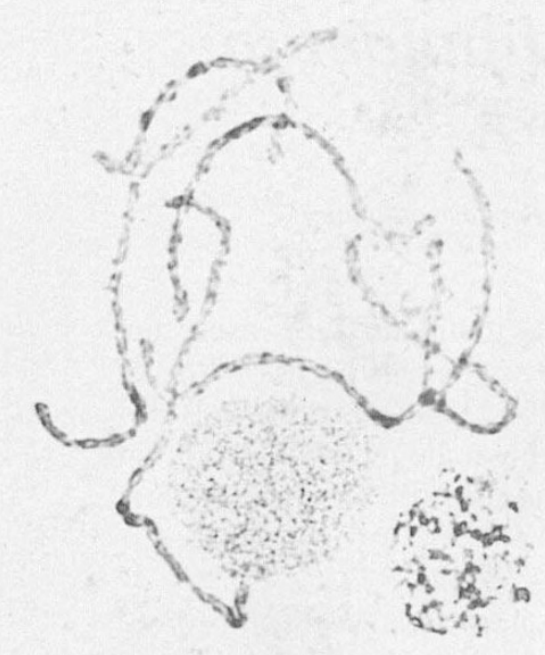

(a)

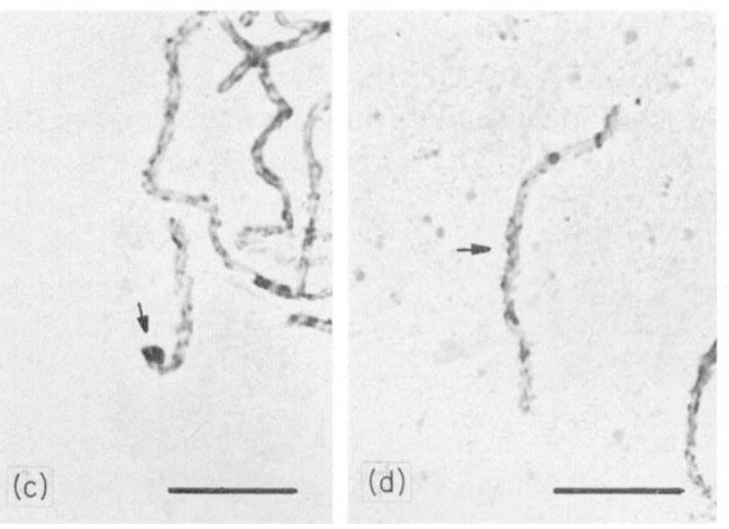

(b)
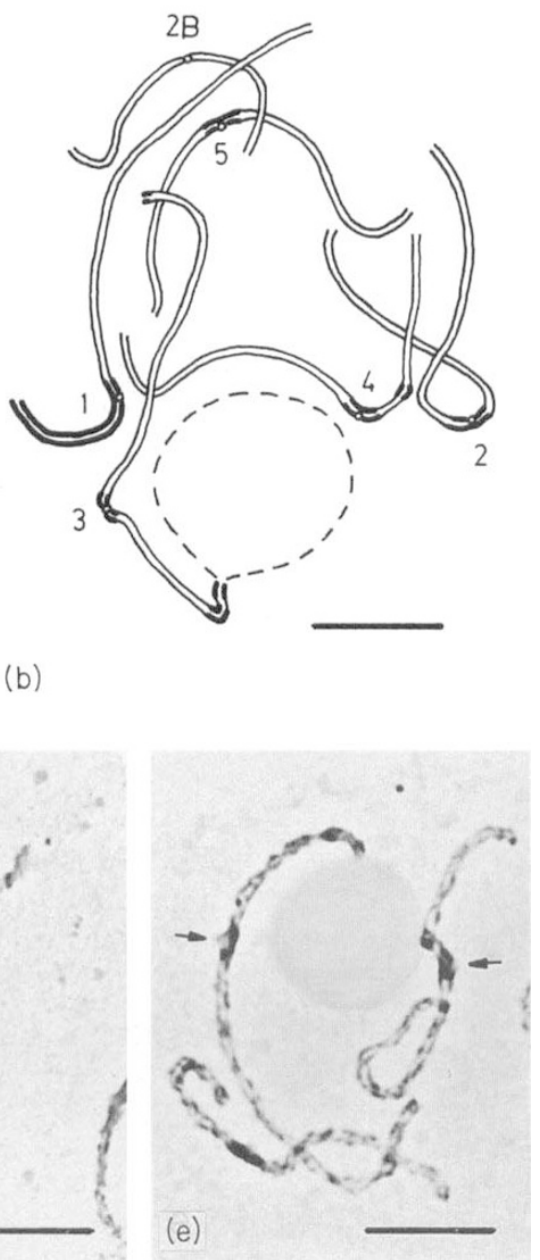

Fig. 1 (a) and (b) photomicrograph and interpretative drawing of the pachytene chromosome complement of S. stipoideum. (c) B-univalent showing inter-arm pairing at pachytene in the $1 \mathrm{~B}$ plant. (d) $2 \mathrm{~B}$ chromosomes showing inter-chromosome pairing at pachytene in the 2B plant. (e) Pachytene stage showing two chromosome bivalents associated with the nucleus: chromosome 3 with a subterminal point of attachment and chromosome 4 with an intercalary point of attachment. Bars equal $10 \mu \mathrm{m}$ in all Figures. Arrows indicate the centromeres.
The morphology and behaviour of the B-chromosomes at pachytene were also studied. When two Bs were present they were always paired to form a bivalent, but did not pair with the A-chromosomes (Figs $1 \mathrm{a}, \mathrm{b}$, and $\mathrm{d}, 4 \mathrm{a}$ and $\mathrm{b})$. The average length of the B-bivalent is $26.00 \pm 1.17 \mu \mathrm{m}$, which is distinctly shorter than any of the A-bivalents. The B-bivalent (Figs 1a, b and $\mathrm{d}$ and 2 ) has an approximately median centromere and is euchromatic along its whole length. The $\mathrm{B}$, when present singly, exhibited inter-arm pairing with its centromere terminal (Fig. 1c). The single B-chromosome did not pair with the standard complement (Figs $1 \mathrm{c}, 3 \mathrm{a}$ and $\mathrm{b})$. The mean value of the $\mathrm{B}$-univalent at pachytene is $13.60 \pm 0.60 \mu \mathrm{m}$ which is about half the length of the B-bivalent. It seems that the B-chromosomes of $S$. stipoideum are all identical in structure, and that they are iso-chromosomes.

The measurements of total length, arm lengths, heterochromatic lengths and arm ratio of the individual $\mathrm{A}-$ and B-bivalents at the pachytene stage are summar- ized in Table 1. An ideogram has been constructed (Fig. 2) incorporating the distinctive features of each chromosome.

\section{The behaviour of B-chromosomes during male meiosis}

The meiotic behaviour of A-chromosomes was found to be normal in plants with and without B-chromosomes. However, the behaviour of the Bs depended upon the number present. In the $1 \mathrm{~B}$ plants, the $\mathrm{B}$ was univalent during diakinesis (Fig. 3a) and metaphase I (Fig. 3b). It divided precociously in the majority of AI cells $(67.4$ per cent). In some cells, the two sister B-chromatids moved to opposite poles so that $\left(\frac{1}{2}-\frac{1}{2}\right)$ distribution resulted (Fig. 3d), and in some others, B-chromatids lagged (Figs $3 e$ and $f$ ). In most of the remaining cells (161 out of 195) the B moved undivided to one pole, giving a chromosome distribu- 
tion of $(1-0)$ (Fig. $3 \mathrm{c})$. In only 34 cells did the undivided $\mathrm{B}$ lag on the equatorial plate. The B-chromosomes which did not divide at anaphase I divided at anaphase II.

At anaphase II, 135 of the 204 dyad groups in the $1 \mathrm{~B}$ plants had lagging B-chromatids, while the remainder had no laggards. Two daughter B-chromatids were distributed to the opposite poles of one dyad and none to the other $\left(\frac{1}{2}-\frac{1}{2}: 0-0\right)$ in 15.2 per cent of cells (Fig. $3 \mathrm{~g}$ ),

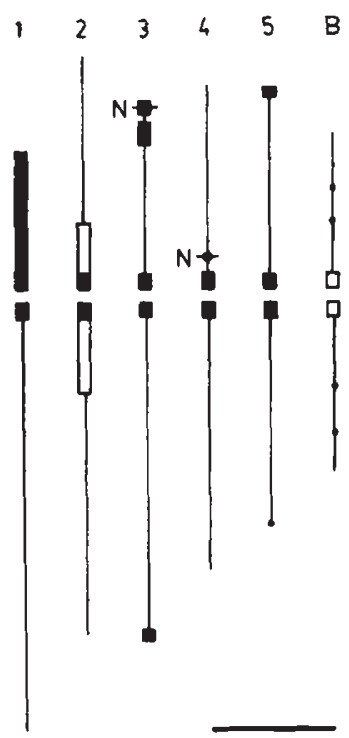

Fig. 2 Pachytene ideograms of the five A-bivalents and the B-bivalent of $S$. stipoideum. Lengths of chromosome arms and heterochromatic regions were obtained from the data presented in Table 1. The spaces in the lines show centromeres. The thin lines represent euchromatic regions, the solid black portions of lines represent heterochromatic regions and barrel shaped knobs, and the hollow portions of lines represent the faintly dark stained segments. Small dots show chromomeres. Letter $\mathrm{N}$ indicates the nucleolus organizing regions. Bar equals $10 \mu \mathrm{m}$. and one-to-one pole of the two dyads $\left(\frac{1}{2}-0: \frac{1}{2}-0\right)$ in 18.6 per cent (Fig. 3h). The former distribution probably resulted from the $(1-0)$ distribution of B-chromosome at anaphase I, while the latter resulted from precocious division at anaphase I. Most tetrads of 1B plants contained micronuclei (72.2 per cent) (Fig. 3i). No micronuclei were found in tetrads of normal plants. Micronuclei must be formed by the eliminated B-chromosomes.

When two B-chromosomes were present, their meiotic behaviour was nearly regular. They were usually associated as bivalents at diakinesis (Fig. 4a) and metaphase I (Fig. 4b). The B-bivalent separation at anaphase I was as regular as A-bivalents with 97.3 per cent of AI cells showing normal disjunction. Consequently a (1-1) distribution was formed (Fig. 4c). Rarely ( 1.7 per cent) the B-bivalent failed to disjoin and moved to one pole, giving a (2-0) distribution (Fig. $4 d)$. Only eight cells $(0.6$ per cent) showed precocious division of B-chromsomes. Out of 1234 AI cells, only five had B laggards (Figs $4 \mathrm{e}$ and $\mathrm{f}$ ). B-chromosome division of the $2 \mathrm{~B}$ plants usually occurred at the second anaphase, giving distribution patterns of $\left(\frac{1}{2}-\frac{1}{2} \cdot \frac{1}{2}-\frac{1}{2}\right)$ in 317 tetrads and $\left(\frac{1}{2}+\frac{1}{2}-\frac{1}{2}+\frac{1}{2}: 0-0\right)$ in only seven. The former pattern (Fig. $4 \mathrm{~g}$ ) has probably arisen from a (1-1) distribution at first anaphase, and the latter from a (2-0) distribution. No B laggards were seen at AII. Examination of 239 tetrads in $2 B$ plants showed only four with micronuclei. The meiotic distributions of the B-chromosomes as described above are summarized in Tables 2 and 3 .

\section{Numerical variation of the B-chromosomes in the germ line and somatic cells}

Fourteen offspring of the 2B female parent were examined cytologically at microsporogenesis. One is an

Table 1 Mean values $(\mu \mathrm{m})$ for pachytene chromosome lengths in Sorghum stipoideum

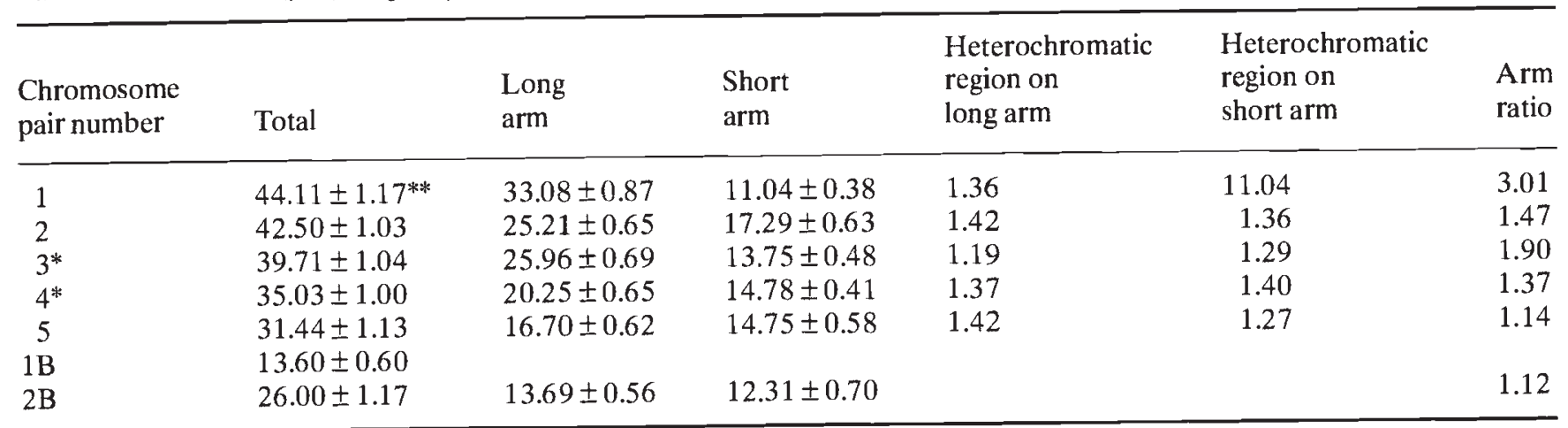

*Nucleolus-organizing chromosome.

** Standard error of the mean. 


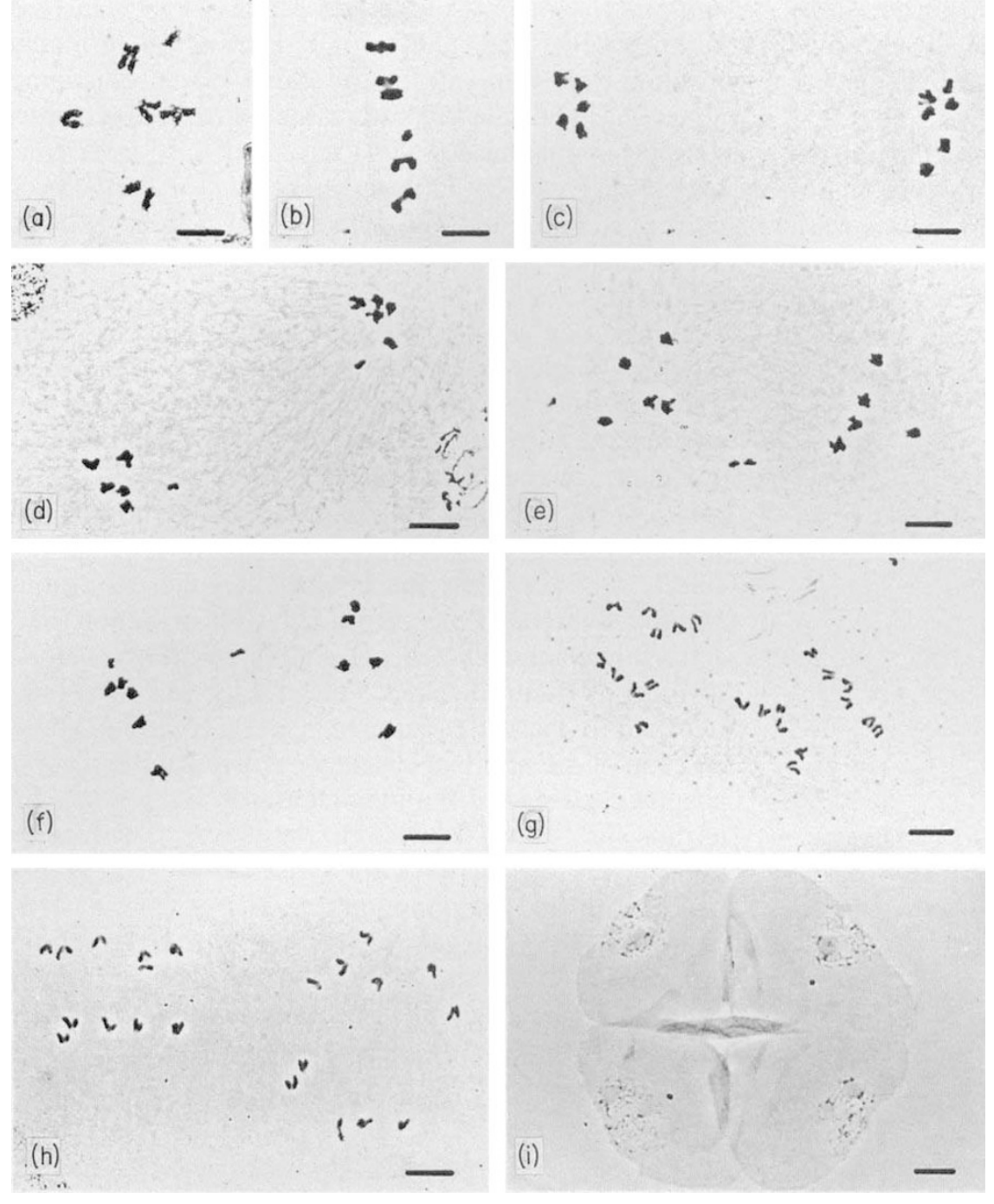

Fig. 3 (a) Diakinesis with five A-bivalents and one B-univalent. (b) Metaphase I with five A-bivalents and one B-univalent. (c) Anaphase I of the IB plant. The B-chromosome has moved to one of the poles. (d) Anaphase I of the $1 \mathrm{~B}$ plant. The B-chromosome has precociously divided into two chromatids and the two members move to the opposite poles. (e) Anaphase I of the $1 \mathrm{~B}$ plant. The B-chromosome divides and lags behind. (f) Anaphase I of the 1B plant. The B-chromosome has precociously divided into two chromatids. One member moves to the pole and the other lags. (g) The $1 \mathrm{~B}$ plant showing $\left(\frac{1}{2}-\frac{1}{2}: 0-0\right)$ distribution of B-chromatids at anaphase II. (h) The IB plant showing $\left(\frac{1}{2}-0: \frac{1}{2}-0\right)$ distribution of $\mathrm{B}$ chromatids at anaphase II. (i) Tetrad group of the IB plant with micronuclei. Bars equal $10 \mu \mathrm{m}$ in all Figures.
$\mathrm{OB}$ plant, while the rest contain B-chromosomes. In nine of these B-containing plants, mosaicism was observed for the presence or absence of B-chromosomes between spikes. Among these nine plants four showed between-spikelet variation in B number. The remaining four plants with Bs had rather uniform B-numbers in their inflorescences. Two of these had $1 \mathrm{~B}$ in nearly all cells, only an extremely low percentage of cells possessed no Bs $(0.5$ per cent $)$. Both of them are regarded as $1 \mathrm{~B}$ plants, and in the other two individuals with $\mathrm{B}$-chromosomes, $2 \mathrm{~B}$ is the predominant number (99 per cent), 0B, 1B and 3B cells could be found in an unusually rare frequency ( 1 per cent). They may be considered as $2 \mathrm{~B}$ plants.

In the somatic cells, the Bs (Figs $4 \mathrm{i}$ and $\mathrm{j}$ ) are clearly distinguishable from the A-chromosomes. The number of B-chromosomes in tapetal cells was unstable and apparently decreasing. During interphase, a micronucleus (Fig. 4k) was occasionally observed in B- containing individuals, but not in normal plants. All cells of stem and leaf meristems in plants with and without B contained only 10 chromosomes (Fig. 4h). B-chromosomes are totally eliminated from these vegetative tissues.

\section{Discussion}

Based on pachytene analysis (Wu, 1980, 1984), the B-chromosomes of Sorghum nitidum and S. purpureosericeum are known to be heterochromatic isochromosomes with the same origin. They probably arose from a nucleolus-organizing chromosome by loss of the long arm after centromere misdivision and subsequent structural change. This opinion is based on the fact that the B-chromosome at pachytene bears a striking resemblance to the short arm of the nucleolusorganizing chromosome. Centromere misdivision and formation of iso-chromosomes is an important origin 
Fig. 4 (a) Diakinesis with five A-bivalents and one B-bivalent. (b) Metaphase I with five A-bivalents and one B-bivalent. (c) The $2 \mathrm{~B}$ plant showing regular segregation of the $2 \mathrm{~B}$ chromosomes at anaphase I. (d) The 2B plant showing non-disjunction of the $2 \mathrm{~B}$ chromosomes at anaphase I. (e) The two B chromosomes laggard behind at anaphase I of the $2 \mathrm{~B}$ plant. (f) The $2 \mathrm{~B}$ plant showing the two divided B-chromosomes laggard behind at anaphase I. (g) The 2B plant showing $\left(\frac{1}{2}-\frac{1}{2}: \frac{1}{2}-\frac{1}{2}\right)$ distribution of B-chromatids at anaphase II. (h) The intercalary meristematic cell of the $1 \mathrm{~B}$ plant at prophase containing no B-chromosome. (i) The tapetal cell of the $1 \mathrm{~B}$ plant at prophase containing a B-chromosome. (j) The tapetal cell of the $2 \mathrm{~B}$ plant at metaphase containing two B-chromosomes. ( $k$ ) A tapetal cell of the $1 \mathrm{~B}$ plant at interphase containing a micronucleus. Bars equal $10 \mu \mathrm{m}$ in all Figures.

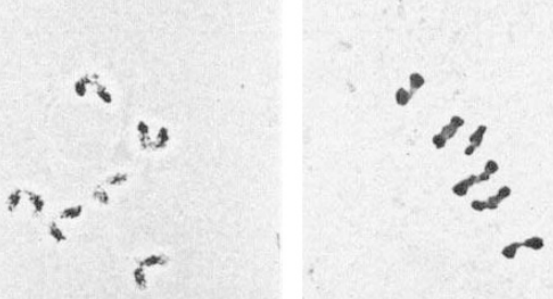

(a)

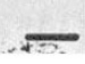

(b)

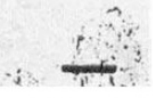

(c)
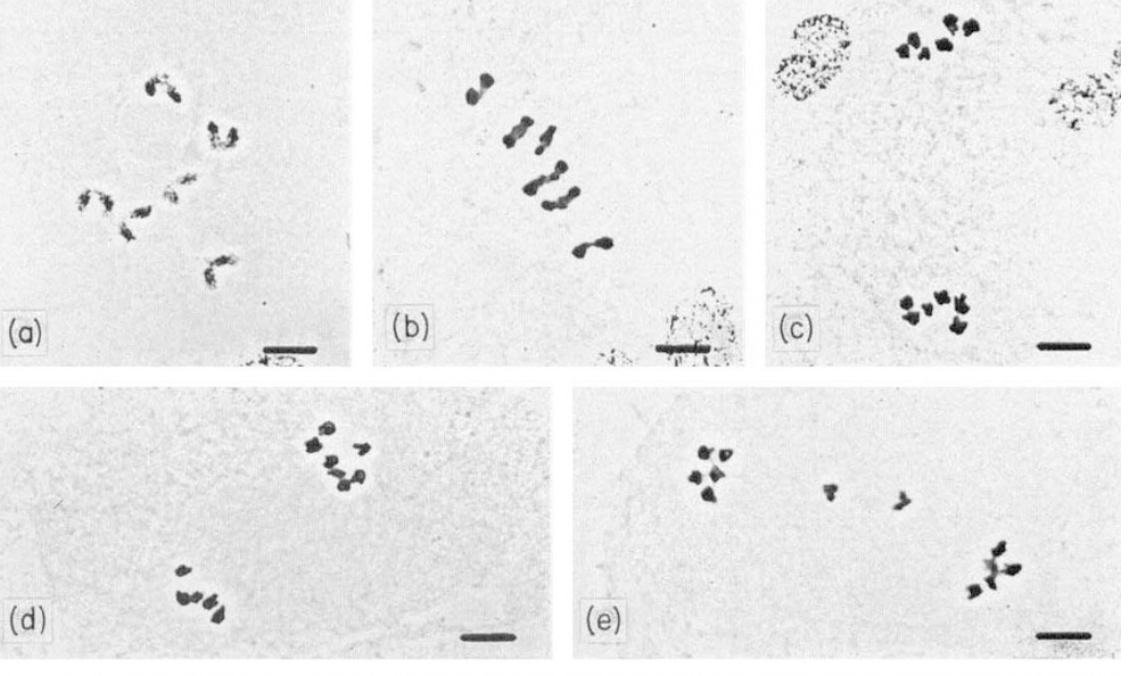

(e)
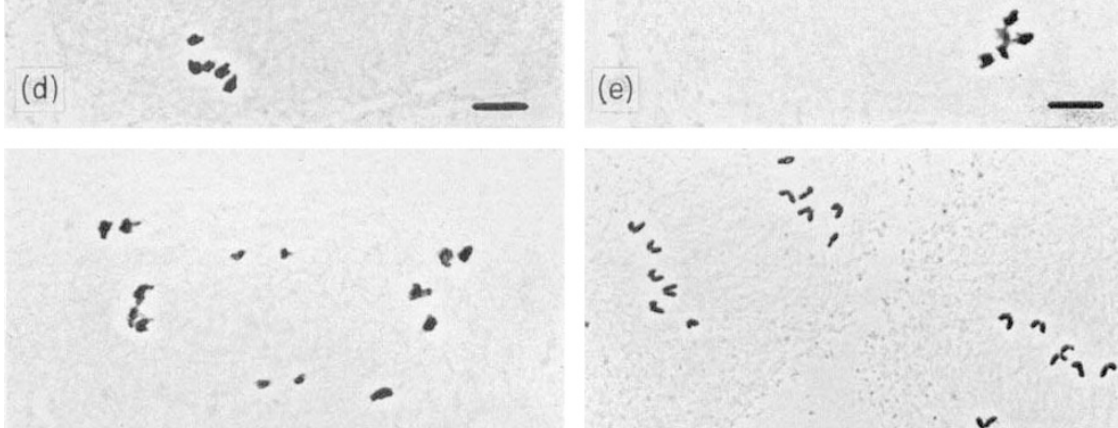

(f)

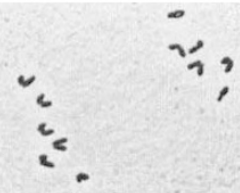

(g)
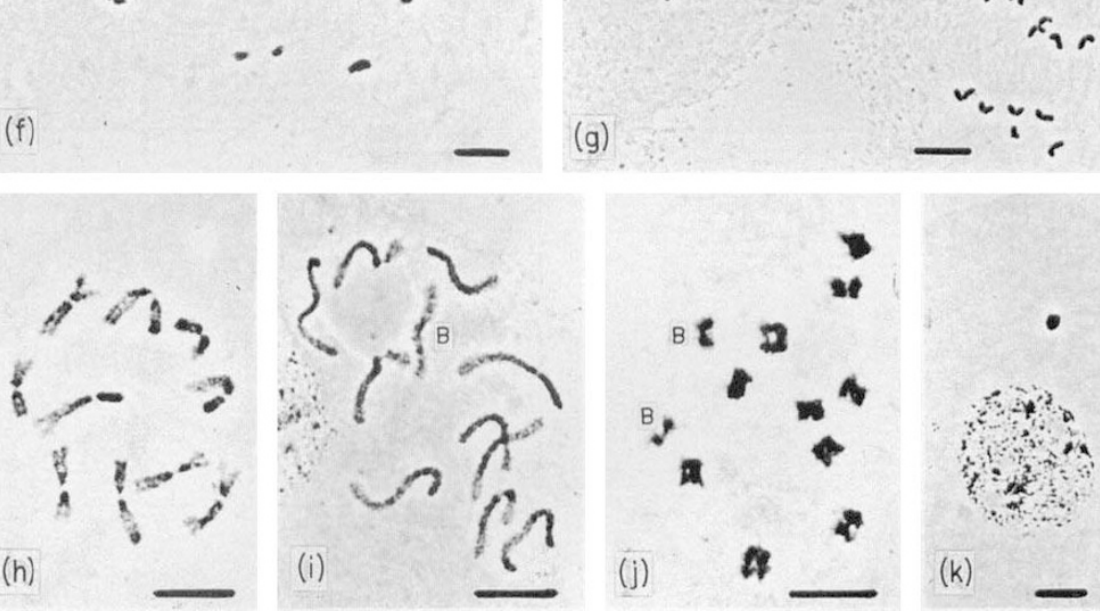

of B-chromosomes (Battaglia, 1964) as proposed for Gasteria (Darlington \& Kefallinou, 1957). It is clear that the B-chromosomes have probably also originated from centromere misdivision of an A-chromosome and subsequent structural changes, but the resemblance between B and the A-chromosome complement of $S$. stipoideum cannot be established.

In the B-containing individuals of $S$. stipoideum, the number of B-chromosomes was not constant in pollen mother cells. Analysis of the mosaicism revealed numerical variation not only between spikes of the same plant but also within spikelets. By contrast, the B-chromosome of Sorghum nitidum was meiotically stable (Wu, 1980). B-chromosomes of different organisms were classified into two categories on the basis of meiotic behaviour by Weimarck (1978). One is represented by Hierochlö̈ (Weimarck, 1978), in which the B-chromosomes frequently show aberrations in centromeric activity. These B-chromosomes usually lag and divide precociously when univalent. They frequently fail to be included in the daughter nuclei and a considerable amount of elimination may occur during male meiosis. The B-chromosomes of $S$. stipoideum fall into this category. The other category is represented by the B-chromosomes of Anthoxanthum and many other grasses (Östergren, 1947; Bosemark, 1957). These B-chromosomes rarely divide into chromatids at anaphase I. They seldom lag or are eliminated during meiosis. A stable system of centromeric activity under univalent conditions seems to have been acquired by 
Table 2 Meiotic behaviour of the B-univalent in the $1 \mathrm{~B}$ plants

\begin{tabular}{lrr}
\hline Distributions of B-univalents at AI & Number of cells observed & Per cent \\
\hline $1-0$ & 161 & 26.92 \\
$\frac{1}{2}-\frac{1}{2}$ & 236 & 39.46 \\
$\frac{1}{2}+\frac{1}{2}-0$ & 2 & 0.33 \\
Laggards & 34 & 5.69 \\
$0-1-0$ & 142 & 23.75 \\
$0-\frac{1}{2}+\frac{1}{2}-0$ & 23 & 3.85 \\
$\frac{1}{2}-\frac{1}{2}-0$ & 598 & \\
Total number of cells & & \\
& Number of dyad groups observed \\
Distributions of B-univalents at AII & 31 & 15.20 \\
$\frac{1}{2}-\frac{1}{2}: 0-0$ & 38 & 18.63 \\
$\frac{1}{2}-0: \frac{1}{2}-0$ & & \\
Laggards & 86 & 42.15 \\
$0-\frac{1}{2}-0: 0-\frac{1}{2}-0$ & 49 & 24.02 \\
$\frac{1}{2}-0: 0-\frac{1}{2}-0$ & 204 & \\
Total number of dyad groups & & \\
\hline
\end{tabular}

Note: $\frac{1}{2}$ shows B chromatid. 1 represents B chromosome.

Table 3 Meiotic behaviour of the B-bivalent in the $2 \mathrm{~B}$ plants

\begin{tabular}{lrr}
\hline Distributions of B-bivalents at AI & Number of cells observed & Per cent \\
\hline $1-1$ & 1201 & 97.33 \\
$2-0$ & 21 & 1.70 \\
$\frac{1}{2}+\frac{1}{2}-\frac{1}{2}+\frac{1}{2}$ & 4 & 0.32 \\
$1-\frac{1}{2}+\frac{1}{2}$ & 2 & 0.16 \\
$1+\frac{1}{2}-\frac{1}{2}$ & 1 & 0.08 \\
Laggards & 2 & 0.16 \\
$0-2-0$ & 2 & 0.16 \\
$1-1-0$ & 1 & 0.08 \\
$0-\frac{1}{2}+\frac{1}{2}, \frac{1}{2}+\frac{1}{2}-0$ & 1234 & \\
Total number of cells & & \\
& Number of dyad groups observed & \\
Distributions of B-bivalents at AII & 317 & 97.84 \\
$\frac{1}{2}-\frac{1}{2}: \frac{1}{2}-\frac{1}{2}$ & 7 & 0 \\
$\frac{1}{2}+\frac{1}{2}-\frac{1}{2}: 0-0$ & 0 & \\
Laggards & 324 & \\
Total number of dyad groups & & \\
\hline
\end{tabular}

Note: $\frac{1}{2}$ shows $B$ chromatid. 1 represents $B$ chromosome.

these B-chromosomes during evolution. The meiotic behaviour of B-chromosomes in S. nitidum (Wu, 1980), place it in the Anthoxanthum class.

The B-chromosome of $S$. stipoideum is variable in number in the microsporocytes, but the number typically decreases in the tapetal tissue, and is zero in the stem and leaf meristems. Complete loss of the B-chromosomes from some tissues has been found in other plants. In Sorghum purpureo-sericeum, Xanthisma texanum and Aegilops speltoides (Darlington \& Thomas, 1941; Berger \& Witkus, 1954; Mendelson \& Zohary, 1972) the B-chromosomes are eliminated from the roots but preserved in shoots and germ cells. Poa alpina has B-chromosomes in primary roots and pollen mother cells, but they are excluded from adventitious roots and leaves (Müntzing \& Nygren, 1955). 
The mechanisms of B-elimination are not known, but may result from non-disjunction and lagging at mitotic anaphase.

\section{Acknowledgments}

The author sincerely thanks Dr J. S. Parker of University of London for valuable suggestions and for critical reading of the manuscript. This work was supported by a grant NSC78-0211-B002-31 from the National Science Council, R.O.C.

\section{References}

battaglia, E. 1964. Cytogenetics of B-chromosomes. Caryologia, 1 7, 245-299.

BERGER, C. A. AND witkus, E. R. 1954. The cytology of Xanthisma texanum DC. I. Differences in the chromosome number of root and shoot. Bull. Torry Bot. Club, 81, 489-491.

BOSEMARK, N. O. 1957. Further studies on accessory chromosomes in grasses. Hereditas, 43, 236-297.
DARLINGTON, C. D. AND Kefallinou, M. 1957. Correlated chromosome aberrations at meiosis in Gasteria. Chromosoma, 8, 364-370.

DARLINGTON, C. D. AND THOMAS, P. T. 1941. Morbid mitosis and the activity of inert chromosomes in Sorghum. Proc. Roy. Soc. London B, 130, 127-250.

JONES, R. N. AND REES, H. 1982. B chromosomes. Academic Press, London.

MENDELSON, D. AND ZOHARY, D. 1972. Behaviour and transmission of supernumerary chromosomes in Aegilops speltoides. Heredity, 29, 329-339.

MUNTZING, A. AND NYGREN, A. 1955. A new diploid variety of Poa alpina with two accessory chromosomes at meiosis. Hereditas 41, 405-422.

OSTERGREN, G. 1947. Heterochromatic B chromosomes in Anthoxanthum. Hereditas, 33, 261-296.

WEIMARCK, G. 1978. Behaviour of B chromosomes in Hierochlö̈ repens (Gramineae) during male meiosis. Hereditas, 88, 7-11.

WU TA-PEN. 1980. Cytogenetic studies of the B chromosomes in Sorghum nitidum. Proc. Nat. Sci. Counc. ROC. 4, 297-306.

WU TA-PEN. 1984. B chromosomes in Sorghum purpureosericeum. Proc. Nat. Sci. Counc, B. ROC. 8, 198-209. 